\title{
"Evaluating Acquisition Time of rfMRI in the Human Connectome Project for Early Psychosis. How much is enough?"
}

\author{
Sylvain Bouix ${ }^{1}$, Sophia Swago ${ }^{1}$, John D. West ${ }^{2}$, Ofer Pasternak ${ }^{1,3}$, Alan Breier ${ }^{4,5}$, \\ Martha E. Shenton ${ }^{1,3,6}$ \\ ${ }^{1}$ Psychiatry Neuroimaging Laboratory, Department of Psychiatry, Brigham and Women's Hos- \\ pital, Harvard Medical School, Boston, MA, USA \\ ${ }^{2}$ Department of Radiology and Imaging Sciences, Center for Neuroimaging, Indiana University \\ School of Medicine, Indianapolis, IN, USA \\ ${ }^{3}$ Department of Radiology, Brigham and Women's Hospital, Harvard Medical School, Boston, \\ MA, USA \\ ${ }^{4}$ Department of Psychiatry, Indiana University School of Medicine, Indianapolis, IN, USA \\ ${ }^{5}$ Larue D. Carter Memorial Hospital, Indianapolis, IN, USA \\ ${ }^{6}$ Clinical Neuroscience Division, Laboratory of Neuroscience, Department of Psychiatry, Vet- \\ erans Affairs (VA) Boston Healthcare System, Harvard Medical School Brockton, MA, USA
}

\begin{abstract}
Resting-state functional MRI (rfMRI) correlates activity across brain regions to identify functional connectivity networks. The Human Connectome Project (HCP) for Early Psychosis has adopted the protocol of the HCP Lifespan Project, which collects 20 min of rfMRI data. However, because it is difficult for psychotic patients to remain in the scanner for long durations, we investigate here the reliability of collecting less than 20 minutes of rfMRI data. Varying durations of data were taken from the full datasets of 11 subjects. Correlation matrices derived from varying amounts of data were compared using the Bhattacharyya distance, and the reliability of functional network ranks was assessed using the Friedman test. We found that correlation matrix reliability improves steeply with longer windows of data up to 11-12 minutes, and $\geq 14$ minutes of data produces correlation matrices within the variability of those produced by 18 minutes of data. The reliability of network connectivity rank increases with increasing durations of data, and qualitatively similar connectivity ranks for $\geq 10$ minutes of data indicates that 10 minutes of data can still capture robust information about network connectivities.
\end{abstract}

Keywords: resting state, acquisition time, Bhattacharyya distance.

\section{Introduction}

Resting-state functional magnetic resonance imaging (rfMRI) can be used to correlate spontaneous fluctuations in the blood oxygen level-dependent (BOLD) signal across regions of the resting brain in order to identify functional connectivity networks [1]. rfMRI is well suited to studying patient populations compared to task-based function-

This is the author's manuscript of the article published in final edited form as:

Bouix, S., Swago, S., West, J. D., Pasternak, O., Breier, A., \& Shenton, M. E. (2017). "Evaluating Acquisition Time of rfMRI in the Human Connectome Project for Early Psychosis. How Much Is Enough?” In Connectomics in NeuroImaging (pp. 108-115). Springer, 
al MRI [2] because it does not require participants to perform any tasks, reducing patient burden and expanding the patient pool to those whose cognitive or physical impairment would otherwise preclude them, and has good signal to noise ratio. rfMRI has potential applications in studying group differences, evaluating treatment, and as a diagnostic biomarker [2]. In recent years, it has increasingly been used to investigate changes in functional networks arising from neurological and psychiatric disorders, including stroke, Alzheimer's disease, depression, and schizophrenia [3].

An open question in rfMRI methodology is how much data to acquire. Typical rfMRI studies only acquire 5-10 minutes of data, but the reliability of estimating functional networks improves with increased scan time. However, there are varying recommendations for how many minutes of data are sufficient. While Van Dijk et al. [4] reported that connectivity estimates stabilized at $\sim 5$ minutes of rfMRI data, Anderson et al. [5] suggested at least 25 minutes be collected, and recently, Laumann et al. [6] saw further gains in reliability with up to 100 minutes of data. The Washington University-University of Minnesota Human Connectome Project (HCP) [7] collected 60 minutes of rfMRI in four runs of 15 minutes. The relatively long scan time was intended to counteract SNR loss due to the high spatial resolution of the data [8].

Our study collects Connectome-like high quality imaging data in an early psychosis population. We have adopted the protocol of the shorter HCP Pilot Lifespan Project [9], which collects $\sim 60$ minutes total of imaging data and includes $\sim 20$ minutes of rfMRI data, to make the scan time more tolerable for patients. However, because it is difficult for psychotic patients to remain in the scanner for long durations, we here investigate the reliability of connectivity estimates from less than 20 minutes of rfMRI data.

\section{Methods}

\subsection{Participants}

Eleven healthy male participants between the ages of 16 and 35 were recruited from Indiana University, Beth Israel Deaconess Medical Center, Massachusetts General Hospital, and McLean Hospital. The Structured Clinical Interview for DSM-5-RV (SCID) [10] was administered to confirm that subjects did not meet criteria for psychiatric diagnosis. All subjects gave written informed consent.

\subsection{Data Acquisition}

Imaging data were collected on two Siemens Prisma 3.0 T MRI scanners at Indiana University and Brigham and Women's Hospital with a 32-channel head coil using a modified protocol based on the HCP LifeSpan Pilot project at the University of Minnesota [9]. Structural, functional, and diffusion data were acquired, with a total scan time of about 70 minutes. Structural data included a T1-weighted image (MPRAGE, $0.8 \mathrm{~mm}^{3}$ isotropic resolution, TR/TE $=2400 / 2.22 \mathrm{~ms}, \mathrm{TI}=1000 \mathrm{~ms}$, flip-angle $=8^{\circ}$ ) and a T2-weighted image (SPACE, $0.8 \mathrm{~mm}^{3}$ isotropic resolution, TR/TE $=3200 / 563 \mathrm{~ms}$ ). Multi-shell diffusion data were acquired four times, twice with anterior-posterior (A- 
P) phase encoding and twice with posterior-anterior (P-A) phase encoding $\left(1.5 \mathrm{~mm}^{3}\right.$ isotropic resolution, 92 directions, $\mathrm{TR} / \mathrm{TE}=3230 / 89.2 \mathrm{~ms}$, multiband acceleration factor $=4, b=1500$ and 3000). rfMRI data were also acquired four times, twice with A$P$ phase encoding and twice with P-A phase encoding (EPI, $2.0 \mathrm{~mm}^{3}$ isotropic resolution, TR/TE $=800 / 37 \mathrm{~ms}$, multiband acceleration factor=8). Each functional run consisted of 420 time points, totaling 5.6 minutes. Subjects were instructed to keep their eyes open during all functional scans. In addition, spin echo field maps were acquired to correct for intensity and geometric distortions.

\subsection{Data Preprocessing}

Structural and functional data were preprocessed with the HCP Minimal Preprocessing Pipelines [11]. The structural pipeline entails registration of the T1w and T2w images, bias field correction, registration to a MNI space template to allow comparison across subjects, reconstruction of white and pial surfaces, and surface registration to a surface atlas. Finally, the data are converted to the Connectivity Informatics Technology Initiative (CIFTI) file format, which combines surface cortical data and volumetric subcortical data in a single "grayordinate" coordinate system.

rfMRI preprocessing includes echo planar imaging (EPI) distortion correction, rfMRI to T1w image registration, motion correction, intensity normalization, and conversion to CIFTI format. The rfMRI data were additionally processed with the Oxford University Centre for Functional MRI of the Brain (FMRIB) group's independent component analysis-based Xnoiseifer - FIX (ICA FIX) [12] pipeline to decompose the BOLD signal and regress out components of physiological noise and noise caused by motion. The resulting time series were then de-meaned and normalized by the standard deviation [13] and concatenated to produce a single time series of 1,680 time points (22.4 minutes).

Contiguous windows of varying durations, ranging from 5.6 to 18 minutes, were randomly extracted from the full 22.4 minute dataset. Durations above 18 minutes were not extracted because the number of different windows that could be obtained would be limited. One hundred windows were extracted for each duration (i.e. 100 of 5.6 minutes, 100 of 6 minutes, etc.). Three a priori parcellation schemes (Fig. 1) were then applied to each window of data: the HCP Nature parcellation (360 regions) [14], Yeo's 17-network parcellation (100 regions), and Yeo's 7-network parcellation (8 regions) [15]. Using Pearson's correlation coefficient, region-to-region functional correlation matrices were generated for all parcellations of each window of data. Using the 17-network parcellation only, the mean within-network connectivities were calculated by averaging the correlations found between regions belonging to the same network. Each network was ranked from lowest to highest mean within-network connectivity. 


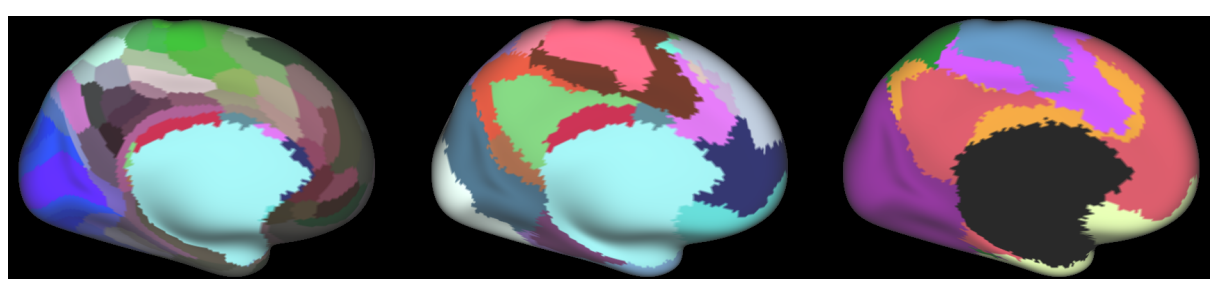

Fig. 1. Different rfMRI parcellation schemes of varying granularity shown on an inflated surface. Left: HCP Nature (360 regions), Center: Yeo 17-network (100 regions), and Right: Yeo 7network (8 regions).

\subsection{Statistical Analysis}

Correlation Matrix Reliability. Each duration of data was directly compared to each of the 18-minute windows within each parcellation to determine matrix reliability. Correlation matrices were directly compared using the Bhattacharyya distance $D_{B}$ [16], which measures the similarity of two probability distributions. The distance $D_{B}$ for two multivariate normal distributions with means $\left(\mu_{1}, \mu_{2}\right)$ and covariance matrices $\left(\Sigma_{1}, \Sigma_{2}\right)$ has the form

$$
D_{B}=\frac{1}{8}\left(\mu_{1}-\mu_{2}\right)^{T} \Sigma^{-1}\left(\mu_{1}-\mu_{2}\right)+\frac{1}{2} \ln \left(\frac{|\Sigma|}{\sqrt{\left|\Sigma_{1}\right|\left|\Sigma_{2}\right|}}\right),
$$

where $\Sigma=\left(\Sigma_{1}+\Sigma_{2}\right) / 2$ and || signifies the matrix determinant. In the current study, $\Sigma_{2}$ was always a correlation matrix derived from an 18-minute window. Because the time series have equal means as a result of de-meaning during preprocessing, Eq. 1 becomes

$$
D_{B}=\frac{1}{2} \ln \left(\frac{|\Sigma|}{\sqrt{\left|\Sigma_{1}\right|\left|\Sigma_{2}\right|}}\right) .
$$

As $\Sigma_{1}$ and $\Sigma_{2}$ become more similar, $D_{B}$ approaches zero.

Network Connectivity Rank Reliability. Networks using the 17-Network parcellation were ranked from lowest to highest within-network connectivity. Changes in network ranks across increasing durations of data were evaluated using the Friedman test, a nonparametric repeated measures analysis, and results were corrected for multiple comparisons.

\section{Results}

\subsection{Correlation Matrix Reliability}

A steep decrease in $D_{B}$, indicating increasingly similar correlation matrices, was observed up to the maximal curvature point observed at durations of $11.21 \pm 0.74$, $11.97 \pm 0.89$, and $12.38 \pm 1.26$ minutes for the HCP Nature, Yeo 17-network, and Yeo-7 network parcellations respectively (Fig. 2). Note that as the number of regions in the 
parcellation decreases, the improvement in matrix similarity is less dramatic. $D_{B}$ then more slowly approached zero. The middle $95 \%$ range of $D_{B}$ values comparing 18 minute windows against other 18-minute windows for each parcellation represents the variability of correlation matrices at the maximum duration. The upper limit of this range was taken as a threshold to determine at which duration correlation matrices became comparable to a matrix derived from 18 minutes. The average durations where $D_{B}$ began to fall within the variability of 18 -minute vs 18 -minute $D_{B}$ values were $14.63 \pm 1.32,14.08 \pm 1.58$, and $13.43 \pm 1.97$ minutes for the HCP Nature, Yeo 17network, and Yeo-7 network parcellations respectively.
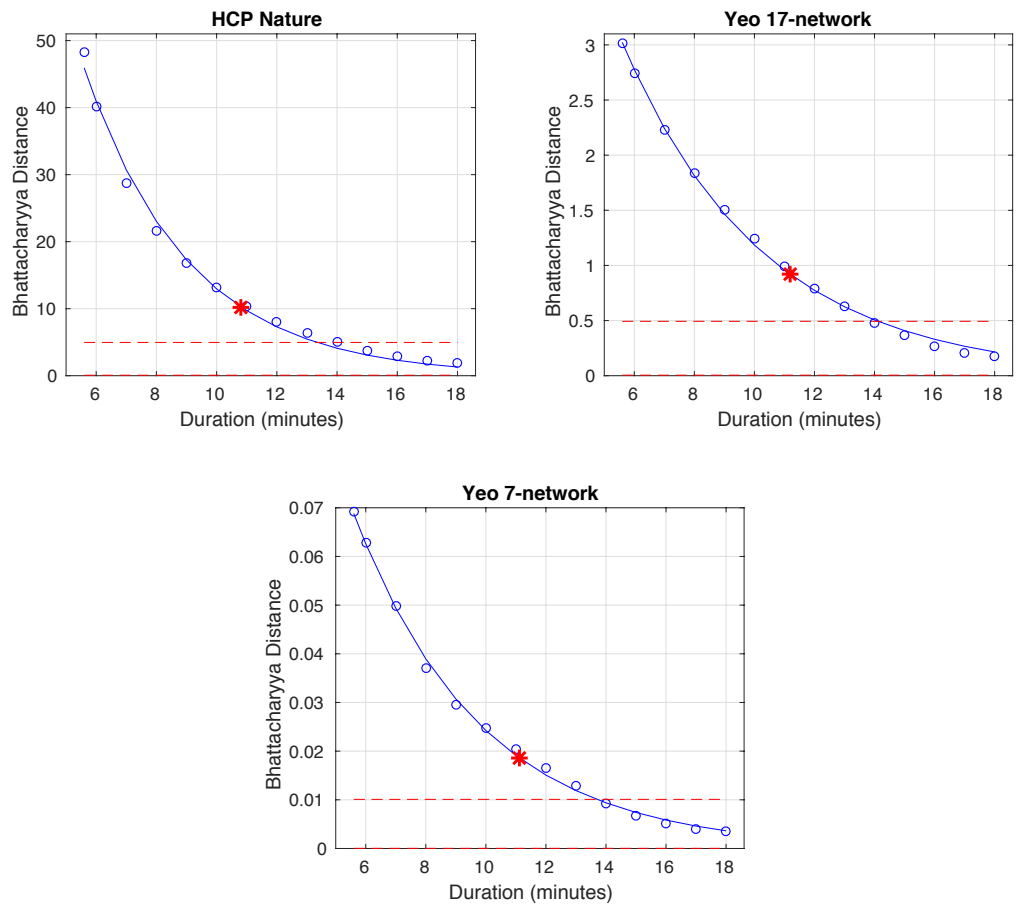

Fig. 2. Mean $D_{B}$ for each duration compared to 18 minutes of data from three parcellations of a representative subject. Points of maximum curvature (star) and middle $95 \%$ ranges of $18 \mathrm{~min}$ vs $18 \min D_{B}$ values (dotted lines) are shown. 

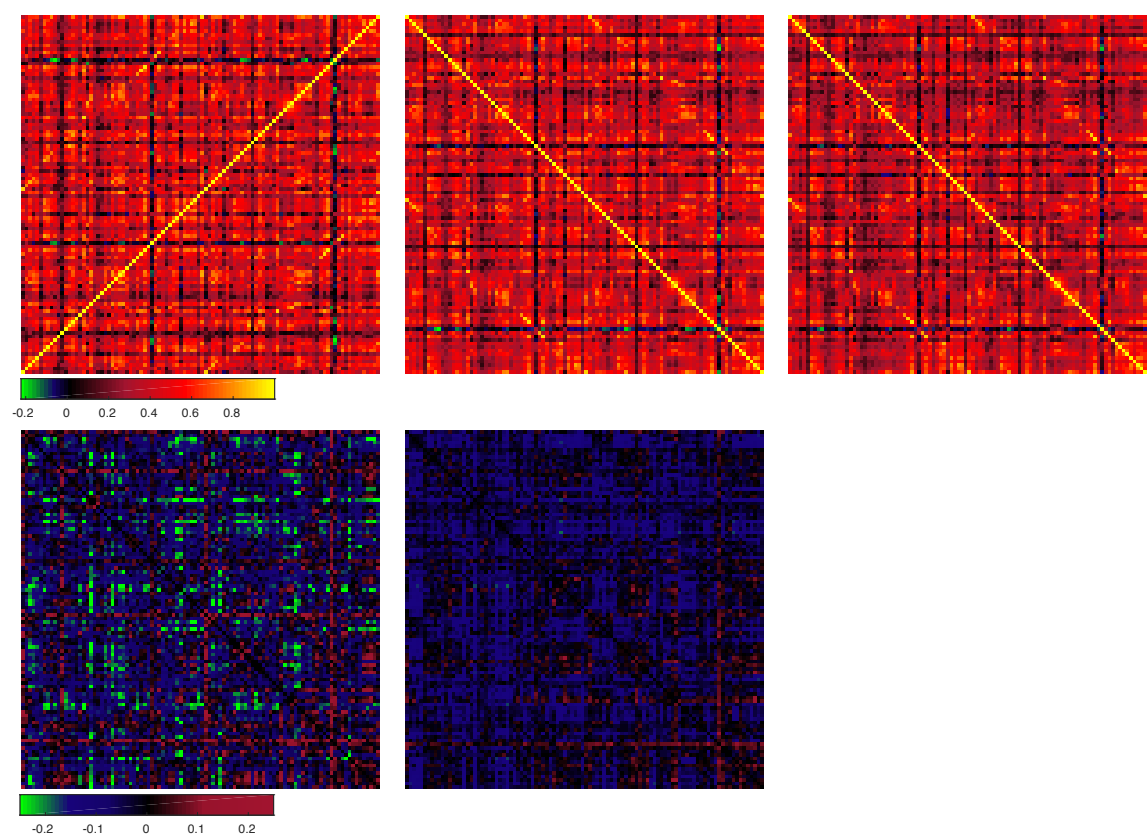

Fig. 3. Top: Representative connectivity matrices derived from 5 minutes (left), 11 minutes (center), and 18 minutes (right) of data using the Yeo 17-network parcellation from a single subject. Bottom: Connectivity differences between 18 and 5 minutes of data (left) and between 18 and 11 minutes of data (right).

\subsection{Network Rank Reliability}

The Friedman test was used to test for a significant difference in mean within-network connectivity rank across varying durations of data. $93.58 \pm 6.68 \%$ of networks had at least one duration that significantly differed in rank $(\mathrm{p}<0.029)$ from of 5.6 to 18 minutes of data. This percentage decreased to $79.14 \pm 6.64 \%$ for rank changes over the range of 10 to 18 minutes, and further to $62.57 \pm 8.43 \%$ from 14 to 18 minutes.

Networks with high within-network connectivity (ranks 16 and 17) were highly reliable even at durations as short as 5-6 minutes (Fig. 4). These ranks primarily corresponded to networks 1 and 2, the peripheral and central visual networks. This agrees with Gonzalez-Castillo, et al. [17] who found the visual network as one of the most temporally stable networks for within-network connections. Many other networks began to display stable rankings at 10-12 minutes of data. 


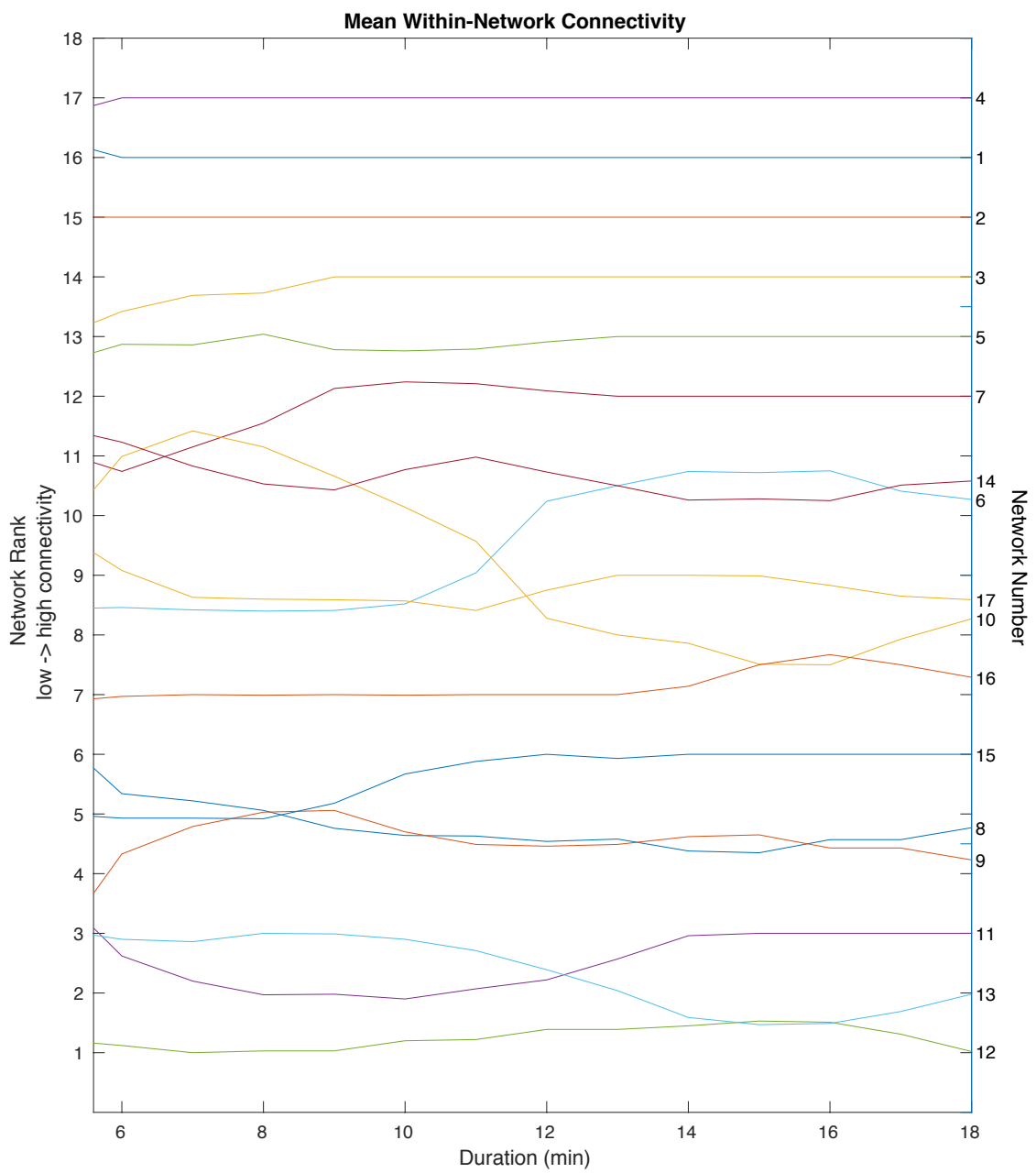

Fig. 4. Mean within-network connectivity ranks from a representative subject using 100 randomly sampled windows of data for each duration.

\section{Discussion and Conclusion}

Using the Bhattacharyya distance, we found that the reliability of functional correlation matrices derived from rfMRI improves monotonically as more data is acquired. The improvement reaches an inflexion point with windows of data around 11-12 minutes, at which point acquiring more data is less beneficial. Fourteen minutes or more of data was found to produce correlation matrices within the variability of those produced by 18 minutes of data. Since all $D_{B}$ values were calculated as a comparison to 18 -minutes of data, these findings are specific to a study whose gold standard is 
$\sim 20$ minutes of rfMRI data. Nonetheless, our method of using the Bhattacharyya distance can be used by other studies to determine how much data is necessary for a session to be usable in further analyses. This is especially valuable for populations that may not tolerate the full scanning protocol of the study.

Our results show that within-network connectivity rank reliability also increases with increasing durations of data, although our statistical tests are not ideally suited for rankings with a zero variance (e.g. a network is always ranked the same in all experiments for a particular duration, as network 4 was for all durations except 5.6 minutes (Fig. 4)). Indeed, we found that many networks qualitatively exhibited similar connectivity ranks starting at 10-12 minutes of data, indicating 10-12 minutes of data may be a suitable amount for many network connectivity analyses. Our results also indicate that the duration required is dependent on the network of interest, as some networks, such as the central and peripheral visual network, are very reliable with as little as 5-6 minutes of data.

Acknowledgments. This research was supported by National Institutes of Health (NIH) grants U01MH109977, R01MH085953, and P41EB015902.

\section{References}

1. Biswal B., Yetkin F.Z., Haughton V.M., Hyde J.S.: Functional connectivity in the motor cortex of resting human brain using echo-planar MRI. Magn Reson Med 34(4), 537-541 (1995).

2. Fox M.D., Greicius M.: Clinical Applications of Resting State Functional Connectivity. Front Syst Neurosci 4, 19 (2010). doi: 10.3389/fnsys.2010.00019

3. Snyder A.Z., Raichle M.E.: A brief history of the resting state: the Washington University perspective. Neuroimage 62(2), 902-910 (2012). doi: 10.1016/j.neuroimage.2012.01.044

4. Van Dijk K.R.A., Hedden T., Venkataraman A., Evans K.C., Lazar S.W., Buckner R.L.: Intrinsic Functional Connectivity As a Tool For Human Connectomics: Theory, Properties, and Optimization. J Neurophysiol 103(1), 297-321 (2010). doi: 10.1152/jn.00783.2009

5. Anderson J.S., Ferguson M.A., Lopez-Larson M., Yurgelun-Todd D.: Reproducibility of Single-Subject Functional Connectivity Measurements. AJNR Am J Neuroradiol 32(3), 548-555 (2011). doi: 10.3174/ajnr.A2330

6. Laumann T.O., Gordon E.M., Adeyemo B., et al.: Functional system and areal organization of a highly sampled individual human brain. Neuron 87(3), 657-670 (2015). doi: 10.1016/j.neuron.2015.06.037

7. Van Essen D.C., Smith S.M., Barch D.M., et al.: The WU-Minn Human Connectome Project: An Overview. NeuroImage 80, 62-79 (2013). doi: 10.1016/j.neuroimage.2013.05.041

8. Smith S.M., Andersson J., Auerbach E.J., et al.: Resting-state fMRI in the Human Connectome Project. NeuroImage 80, 144-168 (2013). doi: 10.1016/j.neuroimage.2013.05.039

9. Phase $1 \mathrm{~b}$ Lifespan Pilot Parameters, http://lifespan.humanconnectome.org/data/phaselbpilot-parameters.html, last accessed 2017/06/09.

10. First M.B., Williams J.B.W., Karg R.S., Spitzer R.L.: Structured Clinical Interview for DSM-5-Research Version (SCID-5 for DSM-5, Research Version; SCID-5-RV). American Psychiatric Association, Arlington, VA (2015). 
11. Glasser M.F., Sotiropoulos S.N., Wilson J.A., et al.: The Minimal Preprocessing Pipelines for the Human Connectome Project. NeuroImage 80, 105-124 (2013). doi: 10.1016/j.neuroimage.2013.04.127

12. Salimi-Khorshidi G., Douaud G., Beckmann C.F., Glasser M.F., Griffanti L., Smith S.M.: Automatic Denoising of Functional MRI Data: Combining Independent Component Analysis and Hierarchical Fusion of Classifiers. NeuroImage 90, 449-468 (2014). doi: 10.1016/j.neuroimage.2013.11.046

13. Beckmann C.F., Smith S.M.: Probabilistic independent component analysis for functional magnetic resonance imaging. IEEE Trans Med Imaging 23(2), 137-152 (2004). doi: 10.1109/TMI.2003.822821

14. Glasser M.F., Coalson T.S., Robinson E.C., et al.: A multi-modal parcellation of human cerebral cortex. Nature 536(7615):171-178 (2016). doi: 10.1038/nature18933

15. Yeo B.T., Krienen F.M., Sepulcre J., et al.: The organization of the human cerebral cortex estimated by intrinsic functional connectivity. J Neurophysiol 106(3), 1125-1165 (2011). doi: $10.1152 /$ jn.00338.2011

16. Bhattacharyya A.: On a measure of divergence between two statistical populations defined by their probability distributions. Bull Calcutta Math Soc 35, 99-109 (1943).

17. Gonzalez-Castillo J., Handwerker D.A., Robinson M.E., et al.: The spatial structure of resting state connectivity stability on the scale of minutes. Front Neurosci 8, 138 (2014). doi: $10.3389 /$ fnins. 2014.00138 